ARTIGO ESPECIAL / SPECIAL ARTICLE

\title{
Mudanças nas condições socioeconômicas e de saúde dos brasileiros durante a pandemia de COVID-19
}

\section{Changes in Brazilians' socioeconomic and health conditions during the COVID-19 pandemic}

\begin{abstract}
Wanessa da Silva de Almeida' (D), Célia Landmann Szwarcwald' (D), Deborah Carvalho Malta" (D), Marilisa Berti de Azevedo Barros III (iD, Paulo Roberto Borges de Souza Júnior' (D), Luiz Otávio Azevedo' (D), Dália Romero' (D), Margareth Guimarães Lima'II' (D), Giseli Nogueira Damacena' (D), Ísis Eloah Machadolv (D), Crizian Saar Gomes ${ }^{\mathrm{V}}$ (D), Maria de Fátima de Pinal (D), Renata Graciel (D), André Oliveira Werneck ${ }^{\mathrm{VI}}$ (D), Danilo Rodrigues Pereira da Silva ${ }^{\mathrm{VII}}$ (iD)
\end{abstract}

RESUMO: Objetivo: Descrever as mudanças nas condições socioeconômicas e de saúde dos brasileiros durante a pandemia de COVID-19. Métodos: Estudo transversal com dados de pesquisa de comportamentos realizada pela internet de 24 de abril a 24 de maio de $2020 \mathrm{com} 45.161$ participantes recrutados por amostragem em cadeia. Foi feita uma análise descritiva de temas abordados na pesquisa: adesão às medidas de restrição social, diagnóstico do novo coronavírus, situação de trabalho e rendimentos, dificuldades nas atividades de rotina, presença de comorbidades, estado de ânimo e acesso aos serviços de saúde. Foram estimados as prevalências e os intervalos de $95 \%$ de confiança. Resultados: Aproximadamente $75 \%$ dos brasileiros aderiram à restrição social. Quanto aos sintomas de gripe, 28,1\% relatou ter apresentado algum sintoma, mas apenas 5,9\% realizou teste para COVID-19. Em relação à situação socioeconômica, 55,1\% relatou diminuição do rendimento familiar, e $7 \%$ ficou sem rendimento; $25,8 \%$ dos indivíduos ficaram sem trabalhar, sendo o grupo de trabalhadores informais o mais afetado (50,6\%). Quanto às condições de saúde, 29,4\% avaliou que a sua saúde piorou; $45 \%$ teve problemas no sono, $40 \%$ apresentou, frequentemente, sentimento de tristeza e $52,5 \%$ de ansiedade/ nervosismo; 21,7\% procurou serviço de saúde e, entre estes, 13,9\% não conseguiu atendimento. Conclusão: Os achados mostram a importância do controle da pandemia de COVID-19 no Brasil, para mitigar os efeitos adversos na situação socioeconômica e nas condições de saúde relacionados às medidas de restrição social.

Palavras-chave: Pandemias. Infecções por coronavírus. Isolamento social. Mudança social. Avaliação em saúde. Brasil.

IInstituto de Comunicação e Informação Científica e Tecnológica em Saúde, Fundação Oswaldo Cruz - Rio de Janeiro (RJ), Brasil. "Departamento de Enfermagem Materno-Infantil e Saúde Pública, Escola de Enfermagem, Universidade Federal de Minas Gerais - Belo Horizonte (MG), Brasil.

"'Departamento de Saúde Coletiva da Faculdade de Ciências Médicas, Universidade Estadual de Campinas - Campinas (SP), Brasil.

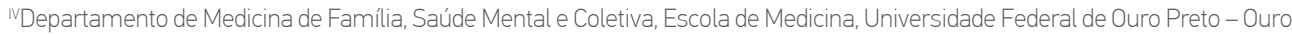
Preto (MG), Brasil.

vDepartamento de Saúde Pública, Faculdade de Medicina, Universidade Federal de Minas Gerais - Belo Horizonte (MG), Brasil. v'Departamento de Nutrição, Escola de Saúde Pública, Universidade de São Paulo - São Paulo (SP), Brasil.

vi'Departamento de Educação Física, Universidade Federal de Sergipe - São Cristóvão (SE), Brasil.

Autor correspondente: Wanessa da Silva de Almeida. Instituto de Comunicação e Informação Científica e Tecnológica em Saúde. Avenida Brasil, 4.365, Pavilhão Haity Moussatché, Manguinhos, CEP: 21040-900, Rio de Janeiro, RJ, Brasil. E-mail: wanessa. almeida@gmail.com

Conflito de interesses: nada a declarar - Fonte de financiamento: nenhuma. 
ABSTRACT: Objective: To describe changes in socioeconomic and health conditions of Brazilians during the COVID19 pandemic. Methodology: Cross-sectional study with data from a web-based behavioral survey carried out from April 24 to May 24, 2020, with 45,161 participants recruited by the chain sampling method. A descriptive analysis of the survey topics was performed: adherence to social restriction measures, diagnosis of the new coronavirus, work situation and income, difficulties in routine activities, presence of comorbidities, psychological issues, and access to health services. Prevalence and respective $95 \%$ confidence intervals were estimated. Results: Approximately $74 \%$ of Brazilians adhered to social restrictions. As for flu symptoms, $28.1 \%$ reported having at least one flu symptom, but only $5.9 \%$ underwent testing for COVID-19. Regarding the socioeconomic impact, $55.1 \%$ reported a decrease in family income, and $7.0 \%$ were left without any income; $25.8 \%$ of the people lost their jobs, with the group of informal workers being the most affected (50.6\%). As for health conditions, $29.4 \%$ reported worsening of health status; $45 \%$, having sleep problems; $40 \%$ frequently presented feelings of sadness, and $52.5 \%$, of anxiety; $21.7 \%$ sought health care, and, among them, $13.9 \%$ did not get care. Conclusion: The findings show the importance of controlling the COVID-19 pandemic in Brazil, to mitigate the adverse effects on the socioeconomic and health conditions related to social restriction measures.

Keywords: Pandemics. Coronavirus infections. Social isolation. Social change. Health evaluation. Brazil.

\section{INTRODUÇÃO}

A ocorrência da COVID-19 causada por uma nova cepa de coronavírus (Sars-CoV-2) foi relatada pela primeira vez na cidade de Wuhan, na China, em dezembro de 2019. Em 30 de janeiro de 2020, a Organização Mundial da Saúde (OMS) declarou o surto de COVID-19 como uma emergência de saúde pública de interesse internacional, e em menos de três meses a doença se espalhou pelo mundo, sendo declarada uma pandemia em 11 de março de $2020^{1-3}$.

No Brasil, o primeiro caso confirmado de COVID-19 foi em 26 de fevereiro de 2020 e, em julho, foram confirmados mais de 2,5 milhões de casos e 90 mil óbitos pelo Sars-CoV-2. Três semanas após o relato do primeiro caso, todos os estados brasileiros já apresentavam pelo menos um caso confirmado da doença ${ }^{4}$.

Diante da ausência de vacinas e de terapias eficazes para o tratamento de COVID-19, uma combinação de medidas de saúde pública tem sido recomendada para mitigar a propagação do vírus e evitar a sobrecarga dos sistemas de saúde ${ }^{5-7}$.

Com a declaração de pandemia e com o crescimento acelerado do número de casos e óbitos no Brasil, medidas de saúde pública na área de controle e prevenção tiveram de ser impostas pelos governos, tais como fechamento de fronteiras, restrição de aulas presenciais, proibição do funcionamento de estabelecimentos comerciais não essenciais, como lojas e restaurantes, além de medidas de distanciamento social e suspensão de reuniões e eventos públicos ${ }^{8}$.

Apesar da indiscutível importância das medidas de restrição social para conter a propagação da doença, são grandes as consequências na sociedade, com efeitos diretos no trabalho e rendimento das famílias e implicações na saúde física e mental dos indivíduos. Além disso, 
os aspectos de incerteza sobre a doença, a separação dos entes queridos e as mudanças nas atividades de rotina trazem, por sua vez, questões psicológicas de relevância ${ }^{9,10}$.

O presente estudo teve os objetivos de descrever a aderência às medidas de restrição social e as mudanças na situação de trabalho e rendimentos nas atividades de rotina e de avaliar o estado de ânimo dos brasileiros durante a pandemia de COVID-19.

\section{MÉTODOS}

Este estudo foi baseado nos dados da ConVid Pesquisa de Comportamentos, realizada entre os dias 24 de abril e 24 de maio de 2020 , por meio do preenchimento de um questionário online. A pesquisa foi elaborada pela Fundação Oswaldo Cruz (FIOCRUZ), em parceria com a Universidade Federal de Minas Gerais (UFMG) e da Universidade Estadual de Campinas (UNICAMP) e obteve aprovação do Comitê de Ética em Pesquisa da Escola Politécnica de Saúde Joaquim Venâncio (Parecer nº 3.980.277) para sua aplicação nacional.

O questionário foi desenvolvido na plataforma Research Electronic Data Capture (RedCap). Todas as informações coletadas foram anônimas, sem possibilidade de identificação dos participantes, e o questionário foi preenchido pelo próprio indivíduo, mediante um telefone celular, computador ou tablet com acesso à internet.

No questionário, foram investigados diversos aspectos, com perguntas relacionadas a características sociodemográficas, infecção pelo novo coronavírus, adesão às medidas de restrição social, estado de saúde, acesso ao serviço de saúde durante a pandemia, estado de ânimo e estilo de vida antes e no decorrer da pandemia ${ }^{11}$.

Os participantes da pesquisa foram recrutados por amostragem em cadeia. Primeiramente, foi selecionado um grupo de pesquisadores de diferentes estados para iniciar a corrente de divulgação. Cada um deles repassou o link da pesquisa para pelo menos 20 pessoas de suas redes sociais, obedecendo a uma estratificação por sexo, faixa de idade e grau de escolaridade, formando a segunda onda da corrente. Então, todos os indivíduos que receberam o link, independentemente da fonte, tiveram a oportunidade de responder ao questionário e de compartilhar o link com outras pessoas de suas redes sociais. Mais detalhes sobre a pesquisa podem ser encontrados no site oficial da ConVid Pesquisa de Comportamentos (https: / / convid.fiocruz.br/).

A base de dados da pesquisa foi calibrada usando as informações da Pesquisa Nacional por Amostra de Domicílios (PNAD) de 2019, por Unidade da Federação, sexo, idade, raça/ cor e grau de escolaridade, para a obtenção de uma amostra com distribuição semelhante à da população brasileira. Antes do início das análises, os dados foram avaliados quanto à presença de duplicidades $(3,6 \%)$ e de dados faltantes $(4,3 \%)$, e ambos foram excluídos da base de dados. A amostra total foi de 45.161 questionários válidos coletados.

Neste estudo, analisou-se parte dos temas investigados na ConVid Pesquisa de Comportamentos. Foram estimadas as prevalências (\%) com os respectivos intervalos de confiança de $95 \%$ (IC95\%), para cada uma das seguintes variáveis: adesão às medidas de restrição social, relato de presença de sintomas de gripe, testagem e infecção pelo novo coronavírus, situação de trabalho 
e rendimento familiar, dificuldades na realização das atividades de rotina, presença de comorbidades de risco para o agravamento da COVID-19, estado de saúde, acesso aos serviços de saúde e estado de ânimo dos indivíduos durante a pandemia.

Para avaliarmos a adesão às medidas de restrição social, foi utilizada a seguinte pergunta: durante a pandemia do novo coronavírus, em que intensidade você fez (ou ainda está fazendo) restrição do contato com as pessoas? As opções de resposta foram: não fiz nada, levei vida normal; procurei tomar cuidados, ficar a distância das pessoas, reduzir um pouco o contato, não visitar idosos, mas continuei trabalhando e saindo; fiquei em casa só saindo para compras em supermercado e farmácia; fiquei rigorosamente em casa, saindo só por necessidade de atendimento à saúde.

Em relação à presença de sintomas de gripe, foi considerada a pergunta: após a chegada da pandemia ao Brasil, apresentou algum sintoma de gripe?, sendo as respostas sim e não. A testagem e o resultado do teste foram avaliados pelas seguintes questões: você fez o teste para saber se estava infectado?; e o resultado do teste foi positivo?, com as seguintes categorias de análise: não fez o teste; fez o teste, com resultado positivo; fez o teste, com resultado negativo; fez o teste, mas não havia recebido o resultado.

Além dessas questões, foi incluída a seguinte pergunta, com respostas sim e não: algum familiar, amigo próximo ou colega de trabalho teve caso grave de doença causada pelo novo coronavírus ou faleceu?

$\mathrm{Na}$ análise da situação de trabalho antes e no decorrer da pandemia, utilizamos as seguintes questões: antes do início da pandemia do novo coronavírus, qual era a sua principal situação de trabalho? (servidor ou trabalhava com carteira assinada; trabalhava sem carteira assinada; trabalhava por conta própria); como a pandemia afetou a sua ocupação/trabalho? (não trabalhava antes e continuou sem trabalhar; continuou trabalhando ou começou a trabalhar durante a pandemia; perdeu o trabalho/ficou sem trabalhar); durante a pandemia, você trabalhou quanto? (menos que o normal; da mesma forma que costumava; mais do que o normal); e, no período da pandemia, que grau de dificuldade você teve para realizar as atividades de trabalho? (nenhum/pouco; moderado; intenso).

Para as classes de rendimento médio domiciliar, fizemos as seguintes questões: antes do início da pandemia do novo coronavírus, qual era a renda total do domicílio?; e no seu domicílio, qual é o número de moradores? Calculou-se a renda média domiciliar per capita, com as seguintes categorias em termos de salário mínimo (SM): até 1/2 SM; 1/2 a um SM; um SM a dois SM; dois SM a quatro SM; quatro SM ou mais.

Além disso, foi possível avaliar o efeito da pandemia na renda das famílias com a seguinte pergunta: como a pandemia afetou a renda da família?, por meio das alternativas: manteve-se a mesma/aumentou; diminuiu um pouco; diminuiu muito; não houve rendimento.

A intensidade do trabalho doméstico foi avaliada pela seguinte questão: a pandemia afetou/modificou a quantidade e o tipo do seu trabalho doméstico? As opções de resposta eram: diminuiu/persistiu igual; aumentou; aumentou muito.

Para a avaliação dos efeitos da pandemia no estado de saúde, foi considerada a seguinte pergunta: Você acha que a pandemia provocou mudanças no seu estado de saúde? As opções de resposta consistiram em: ficou igual/melhorou; piorou.

\section{4}

REV BRAS EPIDEMIOL 2020; 23: E200105 
A presença de comorbidades de risco para o agravamento dos casos de COVID-19 foi baseada no relato de diagnóstico prévio de alguma doença crônica não transmissível (DCNT), como, por exemplo, diabetes, hipertensão, asma/enfisema/doença respiratória crônica ou outra doença do pulmão, doença do coração ou câncer, de acordo com o protocolo de manejo clínico da COVID-19 do Ministério da Saúde ${ }^{12}$.

A procura e o acesso ao serviço de saúde durante a pandemia foram analisados por meio das questões: durante a pandemia do novo coronavírus você procurou atendimento de saúde com um médico, dentista ou outro profissional de saúde? (sim; não); qual foi o principal motivo para você ter procurado atendimento de saúde? (suspeita de COVID-19 ou infecção respiratória; continuação de tratamento ou terapia; problema odontológico; problema de saúde mental; doença ou outro problema de saúde); você conseguiu o atendimento? (sim; não); no período da pandemia, você teve alguma dessas dificuldades relacionadas aos cuidados à sua saúde? (sim; não).

No que se refere ao estado de ânimo, foi avaliada a qualidade do sono por intermédio da questão: a pandemia afetou a qualidade do seu sono? (não afetou / os problemas de sono diminuíram; comecei a ter problemas de sono/os problemas de sono pioraram). Já a frequência de sentimentos de solidão, tristeza ou depressão, ansiedade ou nervosismo, respectivamente, foi medida pelas seguintes perguntas: no período da pandemia, com que frequência você se sentiu isolado(a) dos seus familiares ou amigos próximos? (nunca/poucas vezes; muitas vezes/ sempre); no período da pandemia, com que frequência você se sentiu triste ou deprimido(a)? (nunca/poucas vezes; muitas vezes/sempre); no período da pandemia, com que frequência você se sentiu ansioso(a) ou nervoso(a)? (nunca/poucas vezes; muitas vezes/sempre).

\section{RESULTADOS}

Na Tabela 1, são apresentados os resultados diretamente relacionados à COVID-19. No tocante à adesão às medidas de restrição social, 74,3\% dos indivíduos aderiram às medidas, saindo de casa somente para atividades essenciais, como necessidade de assistência de saúde, farmácias e supermercados. Quanto à presença de algum sintoma de gripe após a chegada da pandemia, 28,1\% relatou ter tido sintomas, e destes apenas 5,9\% haviam realizado teste para saber se estavam infectados pelo novo coronavírus. Entre os testados, 22,5\% (IC95\% 14,5 $33,1)$ apresentaram resultado positivo no teste. Observou-se, ainda, que $17 \%$ dos indivíduos tiveram algum familiar ou amigo próximo com caso grave ou falecimento por COVID-19.

Por meio dos resultados da Tabela 2 sobre a situação do trabalho, constatou-se que $28,9 \%$ não trabalhava antes da pandemia e continuou sem trabalhar, enquanto 50,5\% seguiu trabalhando ou iniciou um novo trabalho durante a pandemia, no entanto $20,6 \%$ perdeu o emprego ou ficou sem trabalhar.

Entre os indivíduos que estavam trabalhando no período analisado, 29,4\% trabalharam mais do que o normal durante a pandemia. A realização de trabalhos domésticos também sofreu mudanças com a pandemia, com $61,7 \%$ da população relatando aumento no desempenho de tais atividades (Tabela 2). 
Tabela 1. Adesão às medidas de restrição social, presença de sintomas e infecção pelo novo coronavírus. ConVid Pesquisa de Comportamentos, 24 de abril a 24 de maio de 2020.

\begin{tabular}{|c|c|c|}
\hline & $\%$ & IC95\% \\
\hline \multicolumn{3}{|l|}{ Adesão às medidas de restrição social durante a pandemia } \\
\hline Não fiz nada, levei vida normal & 1,5 & $1,2-2,0$ \\
\hline $\begin{array}{l}\text { Procurei tomar cuidados, ficar a distância das pessoas, reduzir um pouco o } \\
\text { contato, não visitar idosos, mas continuei trabalhando e saindo }\end{array}$ & 24,2 & $23,0-25,5$ \\
\hline Fiquei em casa, só saindo para compras em supermercado e farmácia & 59,2 & $57,7-60,6$ \\
\hline Fiquei rigorosamente em casa, saindo só por necessidades de atendimento à saúde & 15,1 & $14,1-16,1$ \\
\hline \multicolumn{3}{|l|}{ Relato de sintoma de gripe durante a pandemia } \\
\hline Sim & 28,1 & $26,8-29,4$ \\
\hline Não & 71,9 & $70,6-73,2$ \\
\hline \multicolumn{3}{|l|}{ Realização de teste para COVID-19 entre os que tiveram sintomas de gripe } \\
\hline Não fez o teste & 94,1 & $92,7-95,2$ \\
\hline Fez o teste, com resultado positivo & 1,3 & $0,8-2,1$ \\
\hline Fez o teste, com resultado negativo & 4,0 & $3,1-5,1$ \\
\hline Fez o teste, mas não havia recebido o resultado & 0,6 & $0,4-1,0$ \\
\hline \multicolumn{3}{|l|}{ Familiar ou amigo próximo com caso grave ou falecimento por COVID-19 } \\
\hline Sim & 17,0 & $15,9-18,0$ \\
\hline Não & 83,0 & $82,0-84,1$ \\
\hline
\end{tabular}

IC95\%: intervalo de confiança de 95\%.

Tabela 2. Indicadores relacionados ao trabalho e ao rendimento familiar e dificuldades na realização das atividades de rotina. ConVid Pesquisa de Comportamentos, 24 de abril a 24 de maio de 2020.

\begin{tabular}{|l|c|c}
\hline Situação de trabalho durante a pandemia & $\%$ & IC95\% \\
\hline Não trabalhava antes e continuou sem trabalhar & 28,9 & $27,6-30,2$ \\
\hline Continuou trabalhando ou começou a trabalhar & 50,5 & $49,0-51,9$ \\
\hline Perdeu o trabalho/ficou sem trabalhar & 20,6 & $19,5-21,9$ \\
\hline Intensidade de trabalho durante a pandemia & & \\
\hline Trabalhou menos que o normal & 43,2 & $41,0-45,4$ \\
\hline Trabalhou da mesma forma que costumava & 27,4 & $25,6-29,2$ \\
\hline Trabalhou mais do que o normal & 29,4 & $27,6-31,4$ \\
\hline Grau de dificuldade para realizar as atividades de trabalho durante a pandemia & \\
\hline Nenhum/pouco & 61,4 & $59,3-63,5$ \\
\hline Moderado & 22,2 & $20,5-24,0$ \\
\hline Intenso & 16,4 & $14,8-18,0$ \\
\hline Renda familiar durante a pandemia & & \\
\hline Manteve-se a mesma/aumentou & 37,8 & $36,5-39,2$ \\
\hline Diminuiu um pouco & 30,3 & $29,0-31,7$ \\
\hline Diminuiu muito & 24,8 & $23,5-26,2$ \\
\hline Ficou sem rendimento & 7,0 & $6,3-7,9$ \\
\hline Intensidade do trabalho doméstico durante a pandemia & & \\
\hline Diminuiu/persistiu igual & 38,3 & $36,9-39,8$ \\
\hline Aumentou & 41,5 & $40,1-42,9$ \\
\hline Aumentou muito & 20,2 & $19,1-21,3$ \\
\hline
\end{tabular}

IC95\%: intervalo de confiança de 95\%. 
Em relação à renda familiar, comparando-a com a do período anterior à chegada da pandemia, para 55,1\% houve diminuição na renda, e $7 \%$ ficou totalmente sem rendimentos. $\mathrm{O}$ impacto no rendimento das famílias pode ser observado, igualmente, nos dados apresentados na Figura 1. Na avaliação da renda familiar em categorias de SM, entre os de menor renda (até $1 / 2$ SM per capita), 11,1\% ficou sem rendimento e para $63,5 \%$ houve diminuição da renda. Na categoria superior (quatro SM ou mais per capita), os percentuais foram de 4,3 e $38,4 \%$, respectivamente, ocorrendo um gradiente acentuado por nível de renda: quanto maior a renda familiar, menor a diminuição nos rendimentos (Figura 1).

Além disso, comparando-se a proporção de pessoas que perderam o trabalho após a chegada da pandemia segundo classe de rendimento familiar per capita (Figura 2), constatou-se que os trabalhadores informais (que trabalham por conta própria) nas classes de menor renda foram os mais afetados. No total, entre os que trabalhavam antes da pandemia, 25,8\% ficaram sem trabalhar, enquanto entre os trabalhadores informais esse percentual foi de $50,6 \%$. $\mathrm{Na}$ classe de renda mais pobre, esses percentuais foram de 31,5 e $61 \%$, respectivamente, e na mais rica, de 18,1 e $35,6 \%$.

Quanto aos efeitos da pandemia no estado de saúde dos indivíduos, 29,4\% relataram piora no estado de saúde durante a pandemia. A presença de comorbidades associadas ao agravamento dos casos de COVID-19 foi observada em 33,9\% dos participantes. Sobre a assistência de saúde, $21,7 \%$ procurou por atendimento médico ou odontológico. Entre os que procuraram assistência, $16,5 \%$ precisaram de atendimento por sintomas relacionados à

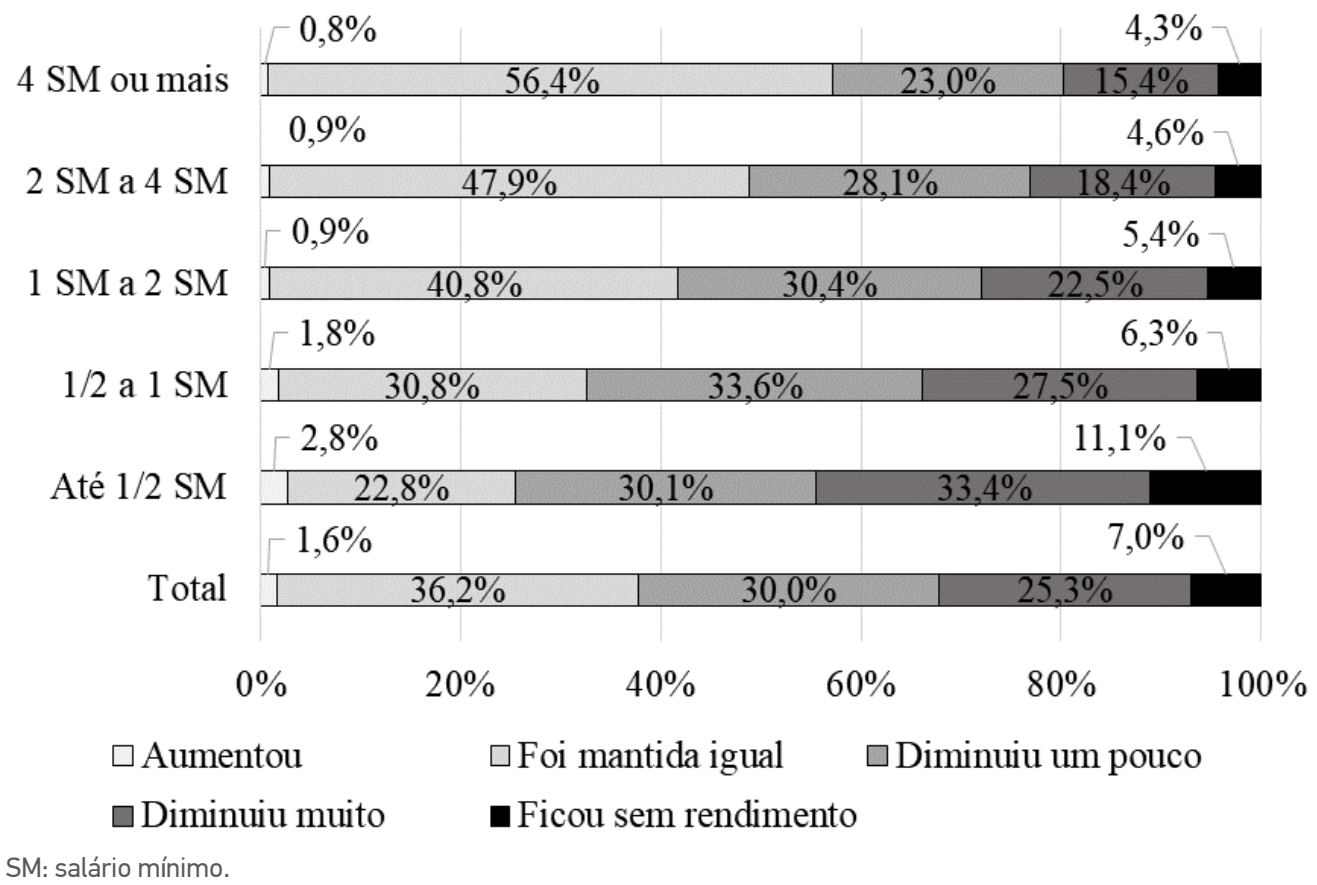

Figura 1. Impacto da pandemia na renda per capita média domiciliar. ConVid Pesquisa de Comportamentos, 24 de abril a 24 de maio de 2020. 


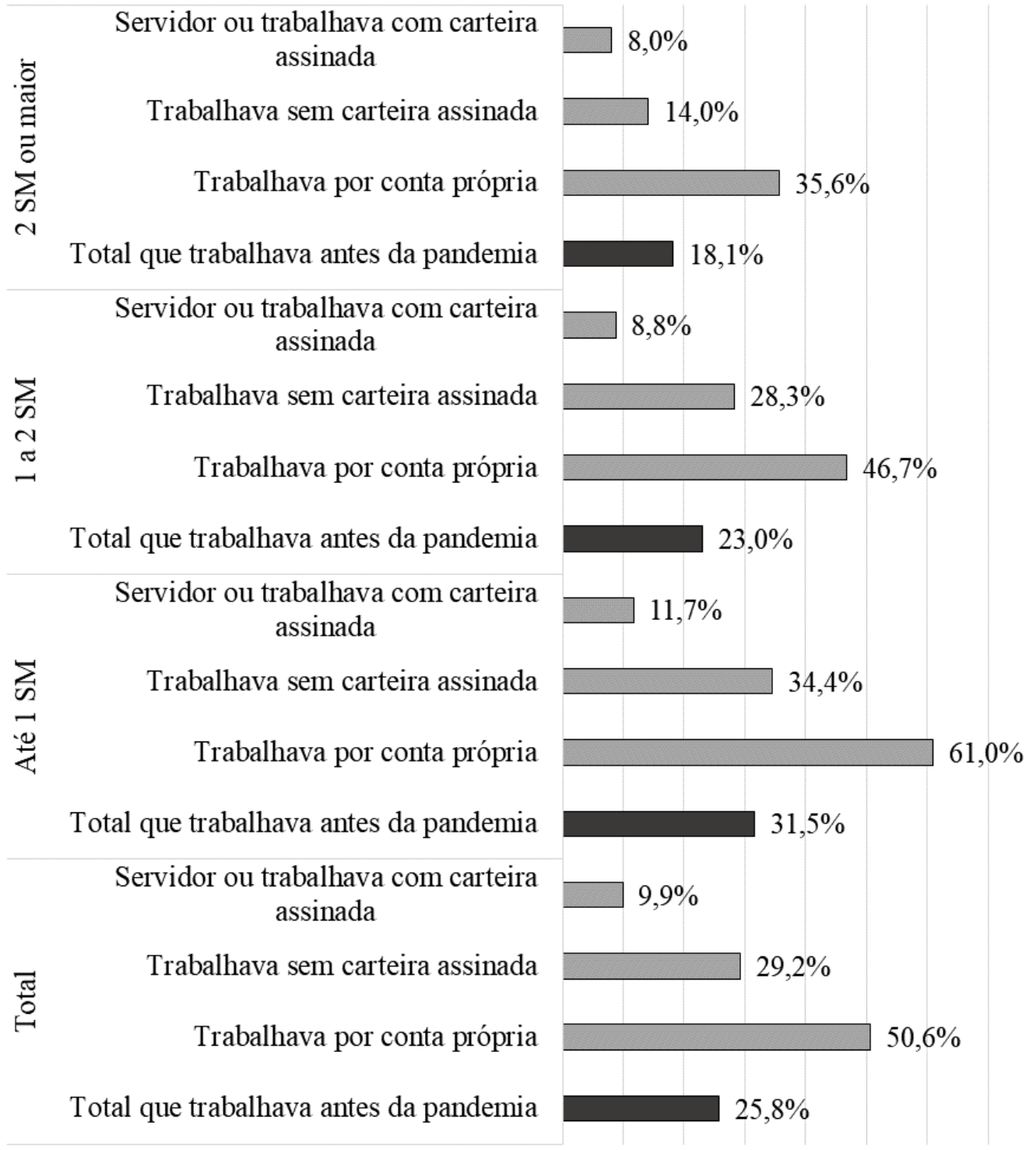

$0 \% 10 \% 20 \% 30 \% 40 \% 50 \% 60 \% 70 \%$

SM: salário mínimo.

Figura 2. Proporção de indivíduos que trabalhavam antes da pandemia e que ficaram sem trabalhar segundo renda média domiciliar per capita e ocupação antes da pandemia. ConVid Pesquisa de Comportamentos, 24 de abril a 24 de maio de 2020.

COVID-19, 25,5\% para continuação de tratamento ou terapia, 19,6\% por problemas odontológicos, 3,4\% por problemas de saúde mental, e 35,1\% por doença ou outros problemas de saúde. Acerca do atendimento, 38,3\% relatou ter tido algum tipo de dificuldade para receber assistência de saúde (Tabela 3). 
Tabela 3. Efeitos no estado de saúde, no acesso aos serviços de saúde e no estado de ânimo. ConVid Pesquisa de Comportamentos, 24 de abril a 24 de maio de 2020.

\begin{tabular}{|c|c|c|}
\hline & $\%$ & IC95\% \\
\hline \multicolumn{3}{|l|}{ Estado de saúde durante a pandemia } \\
\hline Avaliação de piora da saúde & 29,4 & $28,1-30,7$ \\
\hline Presença de comorbidades de risco para COVID-19* & 33,9 & $32,5-35,3$ \\
\hline Procura por atendimento & 21,7 & $20,5-22,8$ \\
\hline \multicolumn{3}{|l|}{ Motivo da procura por atendimento (entre os que procuraram por atendimento) } \\
\hline Sintomas de COVID-19 ou infecção respiratória & 16,5 & $14,1-19,2$ \\
\hline Continuação de tratamento ou terapia & 25,5 & $23,2-28,0$ \\
\hline Problema odontológico & 19,6 & $17,3-22,1$ \\
\hline Problemas de saúde mental & 3,4 & $2,6-4,3$ \\
\hline Doença ou outro problema de saúde & 35,1 & $32,3-38,0$ \\
\hline \multicolumn{3}{|l|}{ Acesso ao serviço de saúde (entre os que procuraram por atendimento) } \\
\hline Conseguiu atendimento & 86,1 & $83,8-88,1$ \\
\hline Teve dificuldades relacionadas aos cuidados à saúde** & 38,3 & $36,9-39,8$ \\
\hline \multicolumn{3}{|l|}{ Problemas no estado de ânimo } \\
\hline Começou a ter problemas no sono ou os problemas no sono pioraram & 44,9 & $43,5-46,3$ \\
\hline Sentiu-se isolado(a) frequentemente & 56,8 & $55,4-58,3$ \\
\hline Sentiu-se triste/deprimido(a) frequentemente & 40,2 & $38,8-41,7$ \\
\hline Sentiu-se ansioso(a)/nervoso(a) frequentemente & 52,5 & $51,1-54,0$ \\
\hline Nenhum desses problemas & 22,8 & $21,6-24,1$ \\
\hline
\end{tabular}

Em relação ao estado de ânimo, 44,9\% começou a ter problemas para dormir ou relatou piora na qualidade do sono após a chegada da pandemia. Mais da metade $(56,8 \%)$ relatou que se sentiu isolada dos familiares e amigos, 40,2\% sentiu-se triste ou deprimido, e 52,5\%, ansioso ou nervoso frequentemente durante a pandemia de COVID-19 (Tabela 3).

\section{DISCUSSÃO}

A ConVid Pesquisa de Comportamentos buscou descrever as mudanças na vida dos brasileiros adultos em diferentes aspectos, durante a pandemia de COVID-19, no Brasil. Sobre a adesão às medidas de restrição social, foi possível observar neste estudo que aproximadamente $75 \%$ dos participantes a fizeram, restringindo o contato com outras pessoas e saindo somente para atividades essenciais. Esses achados corroboram os resultados de outras pesquisas nacionais e internacionais que mostram a boa adesão das medidas por 
parte da população ${ }^{13,14}$, sendo visível o impacto da restrição social na diminuição da taxa de transmissão do novo coronavírus ${ }^{4,15-17}$.

A apresentação de sintomas de gripe durante a pandemia foi relatada por mais de um quarto dos indivíduos de 18 anos ou mais respondentes da pesquisa, mas, entre eles, a proporção de pessoas que foram testadas para saber se estavam infectadas pelo novo coronavírus foi de apenas 5,9\%. Esse percentual pequeno de testagem, principalmente no início da pandemia, também foi observado em outros países ${ }^{18}$. No Brasil, pode ser explicado pela falta de disponibilidade de testes e pelo protocolo de manejo de pacientes, que sugere a testagem obrigatória somente nos casos graves, com sintomas sugestivos de síndrome respiratória aguda grave.

A pandemia de COVID-19 trouxe inúmeros desafios à saúde pública e evidenciou as vulnerabilidades do sistema de saúde para o enfrentamento de emergências ${ }^{1,19}$. Nesse contexto, a disponibilidade de informações oportunas é um desafio recorrente para os tomadores de decisão $\mathrm{o}^{20-22}$. No Brasil, a baixa capacidade de diagnóstico teve implicações importantes na notificação dos casos, que causou não apenas a subestimativa da prevalência na população, mas também a sobre-estimação da taxa de letalidade ${ }^{23}$. Além disso, o fato de pessoas com sintomas mais graves apresentarem maior probabilidade de serem testadas prejudica a análise da situação da doença e o monitoramento das tendências ${ }^{24}$.

A testagem de todos os casos suspeitos é uma das recomendações da OMS para o enfrentamento da COVID-19, na medida em que possibilita o rastreamento dos contatos e o controle da propagação da doença ${ }^{25}$. No entanto, no mês de maio de 2020 , quando foi realizada a pesquisa, ainda se enfrentava a falta de testes de diagnóstico para a doença. Apesar dos investimentos para a produção desses testes e da ampliação dos centros de referência, aumentando a capacidade de processamento de amostras, a distribuição de kits diagnósticos e a disponibilização de testes gratuitos ainda eram deficientes, e os valores cobrados para sua realização no setor privado eram e continuam sendo altos.

No presente estudo, entre os indivíduos que apresentaram sintomas de gripe após a chegada da pandemia e fizeram teste de diagnóstico, o percentual de resultado positivo para COVID-19 foi de 1,3\%. Pesquisa desenvolvida no Rio de Janeiro com doadores de sangue que não tinham sintomas ou histórico anterior conhecido de COVID-19 estimou prevalência de $4 \%{ }^{26}$. A Epicovid19, pesquisa realizada no Rio Grande do Sul, estimou em sua primeira fase prevalência de 4,8\%, aumentando consideravelmente nas demais fases de aplicação. No início da pandemia, nove em cada dez casos não eram notificados ${ }^{23}$.

Os efeitos positivos das medidas de restrição social puderam ser observados na tendência de diminuição das taxas diárias de crescimento do número de casos acumulados no Brasil, da primeira semana de março à última semana de maio de $2020^{27}$. Essas medidas foram essenciais para conter a disseminação da doença e evitar a sobrecarga do sistema de saúde.

Contudo, apesar da importância dessas medidas, no presente estudo, foi possível verificar efeitos adversos na situação de trabalho e no rendimento familiar dos indivíduos. O confinamento domiciliar, bem como o fechamento do comércio não essencial, acarretou demissões e falência de estabelecimentos. Além disso, por causa das alterações na legislação 
trabalhista ${ }^{28}$, a proporção de trabalhadores informais e autônomos — os mais afetados pelas medidas de restrição social — cresceu consideravelmente. Tendo em vista que a diminuição do rendimento acometeu em maior intensidade as famílias com precariedade das condições de vida, pode-se dizer que a pandemia agravou as desigualdades sociais.

A realização de atividades de trabalho também sofreu modificações; um quarto dos trabalhadores passou a desenvolver suas atividades de forma remota. Dessa maneira, o trabalho passou a ocupar e dividir espaço com as outras atividades de rotina e domésticas, e o tempo dedicado ao descanso nem sempre foi suficiente para a reabilitação física e mental ${ }^{29}$.

Embora fundamental para a contenção da pandemia, o distanciamento social trouxe transtornos psíquicos relevantes, relacionados ao sentimento de solidão e isolamento de familiares e amigos, às mudanças no contexto socioeconômico, à falta de controle sobre a própria vida e ao receio de adoecer ${ }^{30,31}$. No Brasil, foram observadas grandes proporções de indivíduos que se sentiram frequentemente isolados, tristes ou deprimidos e ansiosos ou nervosos, bem como de pessoas que relataram problemas no sono. Entre os que apresentaram diagnóstico prévio de depressão, esses efeitos tiveram maior intensidade ${ }^{32}$. Tais resultados estão em consonância com achados de estudos internacionais que avaliaram a saúde mental dos indivíduos durante a pandemia ${ }^{32-36}$.

No que se refere à autoavaliação de saúde, $29,4 \%$ relatou piora no estado de saúde durante a pandemia. Fatores biológicos, como a presença de sintomas de COVID-19 e problemas no estado de ânimo, em conjunto ao contexto de perdas socioeconômicas, afetaram o estado de saúde da população brasileira ${ }^{37}$.

A proporção de pessoas em risco de agravamento dos casos de COVID-19 foi estimada em 33,9\% neste estudo, resultado coerente com o encontrado por Rezende et al. com base nos dados da Pesquisa Nacional de Saúde realizada em 2013, fundamentada, igualmente, na presença de DCNT. Os autores consideraram ainda outro critério, incluindo a obesidade, o fumo e a presença de problemas renais, o que elevou a estimativa para mais da metade da população adulta brasileira sob risco $(54,4 \%)^{38}$.

No que diz respeito à procura por atendimento de saúde durante a pandemia, a maioria dos indivíduos da pesquisa não procurou por serviços de saúde no período analisado. Entre os indivíduos que relataram alguma DCNT preexistente, $25,5 \%$ procuraram atendimento. O esgotamento da capacidade dos serviços e o medo de ser infectado nas unidades de saúde fizeram com que muitos cancelassem suas consultas e deixassem de fazer o acompanhamento dos seus problemas de saúde ${ }^{39}$. Entre os que procuraram atendimento, 16,5\% disseram que o motivo foi a presença de sintomas relacionados à COVID-19 e 38\% apontaram dificuldades no acesso ao serviço de saúde no período analisado.

Neste estudo, apresentaram-se alguns dos aspectos investigados pela ConVid Pesquisa de Comportamentos. Os resultados descritivos da pesquisa estão disponíveis na internet, com acesso livre para a população (https: / / convid.fiocruz.br/). Os resultados foram também divulgados pela mídia, o que possibilitou orientar a população a manter os comportamentos saudáveis e a procurar atendimentos de telemedicina para atenuar os transtornos psicológicos. Diversos artigos relacionados às mudanças nas condições de saúde e no 
estilo de vida durante a pandemia de COVID-19 já foram publicados ou estão aprovados para publicação ${ }^{27,32,37,40,41}$.

A ConVid Pesquisa de Comportamentos apresenta certas limitações referentes ao processo de amostragem em cadeia por redes virtuais. Por ser uma pesquisa pela internet, alguns segmentos populacionais foram sub-representados na amostra, como as pessoas de baixo nível de escolaridade, com dificuldades no manejo de telefones celulares e computadores, ou pessoas sem acesso à internet, que tiveram menor chance de participar. No que se refere à distribuição geográfica da amostra, a pesquisa alcançou todas as Unidades da Federação e aproximadamente 1.700 municípios. Para as variáveis demográficas (sexo, faixa etária e raça), foi obtida a diversidade necessária para ponderação dos dados, de modo a obter representatividade da população brasileira. Apesar da lacuna de pessoas de baixa escolaridade, a amostra grande e abrangente e a calibração dos dados pela PNAD 2019 foram essenciais para a obtenção de uma distribuição amostral semelhante à da população brasileira e para a geração de estimativas adequadas.

\section{REFERÊNCIAS}

1. Steffens I. A hundred days into the coronavirus disease (COVID-19) pandemic. Euro Surveill 2020; 25(14): pii=2000550. https: / / doi.org/10.2807 / 1560-7917. ES.2020.25.14.2000550

2. World Health Organization. Emergency Committee. Statement on the second meeting of the International Health Regulations (2005) Emergency Committee regarding the outbreak of novel coronavirus (2019nCoV). Genebra: World Health Organization; 2020.

3. World Health Organization. Emergency Committee. WHO Director - General's opening remarks at the media briefing on COVID-19 - 11 March 2020. Genebra: World Health Organization; 2020.

4. Fundação Oswaldo Cruz. MonitoraCOVID-19 [Internet]. Rio de Janeiro: Fundação Oswaldo Cruz; 2020 [acessado em 14 jul. 2020]. Disponível em: https: / / bigdata-covid19.icict.fiocruz.br/

5. Salathé M, Althaus CL, Neher R, Stringhini S, Hodcroft E, Fellay J, et al. COVID-19 epidemic in Switzerland: on the importance of testing, contact tracing and isolation. Swiss Med Wkly 2020; 150: w20225. https: / / doi.org/10.4414/smw.2020.20225

6. Mascha EJ, Schober P, Schefold JC, Stueber F, Luedi MM. Staffing with disease-based epidemiologic indices may reduce shortage of intensive care unit staff during the COVID-19 pandemic. Anesth Analg 2020; 131(1): 24-30. https: / / doi.org/10.1213 / ANE.0000000000004849

7. Garcia-AlaminoJM. Aspectos epidemiológicos, clínica y mecanismos de control de la pandemia por Sars-Cov-2: situación en España. Enferm Clin 2020. https: / / doi. org/10.1016/j.enfcli.2020.05.001

8. Aquino EML, Silveira IH, Pescarini JM, Aquino R, Souza-Filho JA, Rocha AS, et al. Social distancing measures to control the COVID-19 pandemic: potential impacts and challenges in Brazil. Ciên Saúde Colet 2020; 25(Supl. 1): 2423-46. https: / / doi. org/10.1590/1413-81232020256.1.10502020

9. Brooks SK, Webster RK, Smith LE, Woodland L, Wessely S, Greenberg N, et al. The psychological impact of quarantine and how to reduce it: rapid review of the evidence. Lancet 2020; 395(10227): 91220. https: / / doi.org/10.1016/S0140-6736(20)30460-8

10. Lima CT, Carvalho PMM, Lima IAAS, Nunes JVAO, Saraiva JS, Souza RI, et al. The emotional impact of Coronavirus 2019-nCoV (new Coronavirus disease). Psychiatry Res 2020; 287: 112915. https:// doi. org/10.1016/j.psychres.2020.112915

11. Instituto de Comunicação e Informação Científica e Tecnológica em Saúde. ConVid - Pesquisa de Comportamentos [Internet]. 2020 [acessado em 16 jul. 2020]. Disponível em: https:// doi.org/10.7303/ syn22250673.1

12. Brasil. Ministério da Saúde. Secretaria de Atenção Especializada à Saúde. Departamento de Atenção Hospitalar, Domiciliar e de Urgência. Protocolo de manejo clínico da COVID-19 na Atenção Especializada [Internet]. Brasília: Ministério da Saúde; 2020 [acessado em 16 jul. 2020]. 48 p. Disponível em: http:/ / bvsms. 
saude.gov.br/bvs/publicacoes/manejo_clinico_covid19_atencao_especializada.pdf

13. Barros AJD, Victora CG, Menezes AMB, Horta BL, Hartwig F, Victora G, et al. Social distancing patterns in nine municipalities of Rio Grande do Sul, Brazil: the Epicovid19/RS study. Rev Saúde Pública 2020; 54: 75. https://doi.org/10.11606/s1518-8787.2020054002810

14. Webster RK, Brooks SK, Smith LE, Woodland L, Wessely S, Rubin GJ. How to improve adherence with quarantine: rapid review of the evidence. Public Health 2020; 182: 163-9. https://doi.org/10.1016/j. puhe.2020.03.007

15. Qiu Y, Chen X, Shi W. Impacts of social and economic factors on the transmission of coronavirus disease 2019 (COVID-19) in China. J Popul Econ 2020; 33: 1127-72. https: / doi.org/10.1007/ s00148-020-00778-2

16. Shen M, Peng Z, Guo Y, Rong L, Li Y, Xiao Y, et al. Assessing the Effects of Metropolitan-Wide Quarantine on the Spread of COVID-19 in Public Space and Households. Int J Infect Dis 2020; 96: 503-5. https: / / doi.org/10.1016/j.ijid.2020.05.019

17. Tang B, Xia F, Tang S, Bragazzi NL, Li Q, Sun X, et al. The effectiveness of quarantine and isolation determine the trend of the COVID-19 epidemics in the final phase of the current outbreak in China. Int J Infect Dis 2020; 95: 288-293. https: / doi.org/10.1016/j.ijid.2020.03.018

18. Li R, Pei S, Chen B, Song Y, Zhang T, Yang W, et al. Substantial undocumented infection facilitates the rapid dissemination of novel coronavirus (SARSCoV-2). Science 2020; 368(6490): 489-93. https: / / doi. org/10.1126/science.abb3221

19. Reis RF, Quintela BM, Campos JO, Gomes JM, Rocha BM, Lobosco M, et al. Characterization of the COVID19 pandemic and the impact of uncertainties, mitigation strategies, and underreporting of cases in South Korea, Italy, and Brazil. Chaos Solitons Fractals 2020; 136: 109888. https: / / doi.org/10.1016/j.chaos.2020.109888

20. Barreto ML, Barros AJD, Carvalho MS, Codeço CT, Hallal PRC, Medronho RA, et al. O que é urgente e necessário para subsidiar as políticas de enfrentamento da pandemia de COVID-19 no Brasil? Rev Bras Epidemiol 2020; 23: e200032. https://doi. org/10.1590/1980-549720200032

21. Prado MFD, Antunes BBP, Bastos LDSL, Peres IT, Silva AABD, Dantas LF, et al. Analysis of COVID-19 underreporting in Brazil. Rev Bras Ter Intensiva 2020; 32(2): 224-8. https: / / doi.org/10.5935/0103-507x.20200030

22. Hallal PC, Horta BL, Barros AJD, Dellagostin OA, Hartwig FP, Pellanda LC, et al. Trends in the prevalence of COVID-19 infection in Rio Grande do Sul, Brazil: repeated serological surveys. Ciên Saúde Colet 2020; 25(Supl. 1): 2395-401. https: / / doi. org/10.1590/1413-81232020256.1.09632020
23. Silveira MF, Barros AJD, Horta BL, Pellandra LC, Victora GD, Dellagostin OA, et al. Population-based surveys of antibodies against SARS-CoV-2 in Southern Brazil. Nat Med 2020; 26: 1196-9. https: / / doi.org/10.1038/s41591-020-0992-3

24. Lau H, Khosrawipour T, Kocbach P, Ichii H, Bania J, Khosrawipour V. Evaluating the massive underreporting and undertesting of COVID-19 cases in multiple global epicenters. Pulmonology 2020; 1-6. https:/ / doi.org/10.1016/j.pulmoe.2020.05.015

25. World Health Organization. Considerations in adjusting public health and social measures in the context of COVID-19 - Interim guidance - 16 April 2020. Genebra: World Health Organization; 2020.

26. Amorim Filho L, Szwarcwald CL, Mateos SOG, Ponce de Leon ACM, Medronho RA, Veloso VG, et al. Seroprevalence of anti-SARS-CoV-2 among blood donors in Rio de Janeiro, Brazil. Rev Saúde Pública 2020; 54: 69. http:// doi.org/10.11606/ s1518-8787.2020054002643

27. Szwarcwald CL, Souza Júnior PRB, Malta DC, Barros MBA, Magalhães MAFM, Xavier DR, et al. Adesão às medidas de restrição social e efeitos sobre a disseminação covid-19 no Brasil. Epidemiol Serv Saúde 2020. No prelo.

28. Brasil. Lei $\mathrm{n}^{\circ}$ 13.467, de 13 de julho de 2017. Altera a Consolidação das Leis do Trabalho (CLT), aprovada pelo Decreto-Lei $n^{\circ} 5.452$, de $1^{\circ}$ de maio de 1943 , e as Leis $\mathrm{n}^{\circ}$ 6.019, de 3 de janeiro de 1974, 8.036, de 11 de maio de 1990, e 8.212, de 24 de julho de 1991, a fim de adequar a legislação às novas relações de trabalho [Internet]. 2017 [acessado em 7 ago. 2020]. Disponível em: http: / /www.planalto.gov.br/ccivil_03/_ato20152018/2017/lei/113467.htm

29. Losekann RGCB, Mourão HC. Desafios do teletrabalho na pandemia covid-19: quando o home vira office. Cad Adm 2020; 28: 71-5. https:// doi.org/10.4025/ cadadm.v28i0.53637

30. Moretti SA, Guedes-Neta ML, Batista EC. Nossas Vidas em Meio à Pandemia da COVID - 19: Incertezas e Medos Sociais. Rev Enfermagem e Saúde Coletiva 2020; 4(2): 32-41.

31. Lima SO, Silva MA, Santos MLD, Moura AMM, Sales LGD, Menezes LHS, et al. Impactos no comportamento e na saúde mental de grupos vulneráveis em época de enfrentamento da infecção COVID-19: revisão narrativa. Rev Eletrônica Acervo Saúde 2020; (46): e4006. https: / / doi.org/10.25248/ reas.e4006.2020

32. Barros MBA, Lima MG, Malta DC, Szwarcwald CL, Azevedo RCS, Romero D, et al. Relato de tristeza/ depressão, nervosismo/ansiedade e problemas de sono na população adulta brasileira durante a pandemia de COVID-19. Epidemol Serv Saúde 2020; 29(4): e2020427. http:/ / doi.org/10.1590/s1679-49742020000400018 
33. Huang Y, Zhao N. Generalized anxiety disorder, depressive symptoms and sleep quality during COVID19 outbreak in China: a web-based cross-sectional survey. Psychiatry Res 2020; 288: 112954. https: / / doi. org/10.1016/j.psychres.2020.112954

34. Guo Y, Cheng C, Zeng Y, Li Y, Zhu M, Yang W, et al. Mental health disorders and associated risk factors in quarantined adults during the COVID-19 outbreak in China: a cross-sectional study. J Med Internet Res 2020; 22(8): e20328. https: / / doi.org/10.2196/20328

35. Rossi R, Socci V, Talevi D, Mensi S, Niolu C, Pacitti F, et al. COVID-19 Pandemic and Lockdown Measures Impact on Mental Health Among the General Population in Italy. Front Psychiatry 2020; 11: 790. https: / / doi.org/10.3389/fpsyt.2020.00790

36. Gallagher MW, Zvolensky MJ, Long LJ, Rogers AH, Garey L. The Impact of COVID-19 Experiences and Associated Stress on Anxiety, Depression, and Functional Impairment in American Adults. Cogn Ther Res 2020. https: / / doi.org/10.1007/s10608-020-10143-y

37. Szwarcwald CL, Damacena GN, Barros MBA, Malta DC, Souza Júnior PRB, Azevedo LO, et al. Factors affecting the Brazilians' self-rated health during the COVID-19 pandemic. Cad Saúde Pública 2020. No prelo.

38. Rezende LFM, Thome B, Schveitzer MC, Souza-Júnior PRB, Szwarcwald CL. Adults at high-risk of severe coronavirus disease-2019 (COVID-19) in Brazil. Rev Saúde Pública 2020; 54: 50. https: / / doi.org/10.11606/ s1518-8787.2020054002596

39. Emanuel EJ, Persad G, Upshur R, Thome B, Parker M, Glickman A, et al. Fair allocation of scarce medical resources in the time of COVID-19. N Engl J Med 2020; 382: 2049-55. https:// doi.org/10.1056/NEJMsb2005114

40. Malta DC, Szwarcwald CL, Barros MBA, Gomes CS, Machado IE, Souza Júnior PRB, et al. A pandemia da COVID-19 e as mudanças no estilo de vida dos brasileiros adultos: um estudo transversal, 2020. Epidemiol Serv Saúde 2020; 29(4): e2020407. https: / / doi.org/10.1590/S1679-49742020000400026

41. Werneck AO, Silva DR, Malta DC, Souza-Júnior PRB, Azevedo LO, Barros MBA, et al. Lifestyle behaviors changes during the COVID-19 pandemic quarantine among 6,881 Brazilian adults with depression and 35,143 without depression. Ciênc Saúde Colet 2020; 25(Supl. 2): 4151-6. http:/ / doi. org/10.1590/1413-812320202510.2.27862020

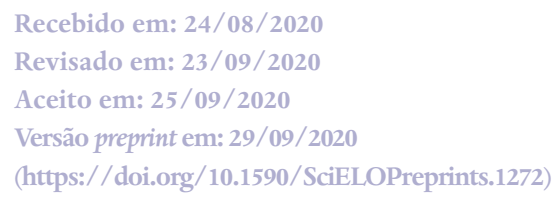

Contribuição dos autores: Almeida WS e Szwarcwald CL contribuíram com a concepção, análise dos dados e elaboração do texto. Malta DC e Barros MBA contribuíram com a elaboração do texto e revisão crítica do conteúdo. Souza Júnior PRB, Azevedo LO, Romero D, Lima MG, Damacena GN, Machado IE, Gomes CS, Pina MF, Gracie R, Werneck AO e Silva DRP contribuíram com a elaboração do texto e revisão final. Todos os autores aprovaram a versão final do manuscrito. 